\title{
Closure of gastric incision with a barbed suture after needlescope-assisted hybrid NOTES
}
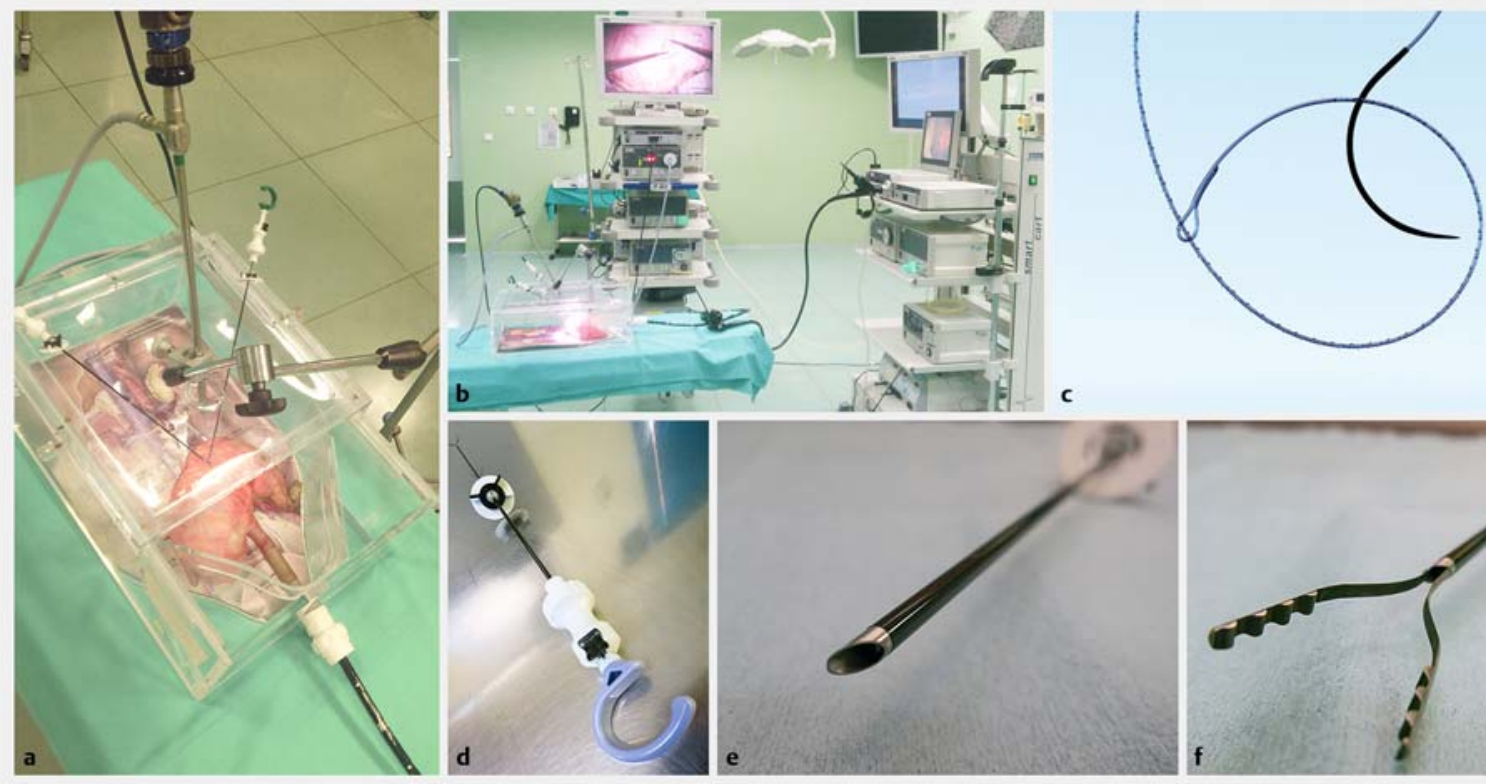

- Fig. 1 Needlescope-assisted hybrid natural orifice transluminal endoscopic surgery (NOTES) and closure of the gastric incision: placement of the instruments and equipment, and a detailed view of the barbed suture (V-Loc) and needlescopic forceps (MiniLap).

Provision of safe peritoneal access without organ injury and guaranteeing secure transluminal closure are two challenging goals in natural orifice transluminal endoscopic surgery (NOTES) [1]. Minilaparoscopically assisted natural orifice surgery (MANOS) is a hybrid NOTES technique that could enable performance of more complex procedures and that can help to avoid the disadvantages of a purely NOTES approach, while minimizing the invasiveness of the laparoscopic procedure [2].

This is a preliminary ex vivo study of a gastrotomy closure method - safe scarless gastric laparoscopic closure (SSGLC) - carried out during a modified MANOS procedure in porcine stomach ( $\downarrow$ Video 1). For this technique, two percutaneous needlescopic instruments (MiniLap Clutch Grasper; Teleflex, Morrisville, North Carolina, USA) were combined with the rigid laparoscopic optics ( $\triangleright$ Fig.1). Under laparoendoscopic guid- ance, the penetrating tip of the MiniLap was inserted into the previously insufflated gastric lumen and used to grasp the guidewire of the balloon dilator, pulling it through the gastric wall and ensuring safe entry of the gastroscope ( $\triangleright$ Fig. 2 ). For gastrotomy closure we used the knotless, self-anchoring surgical suture V-Loc (Medtronic/Covidien; Minneapolis, Minnesota, USA), introduced percuta-

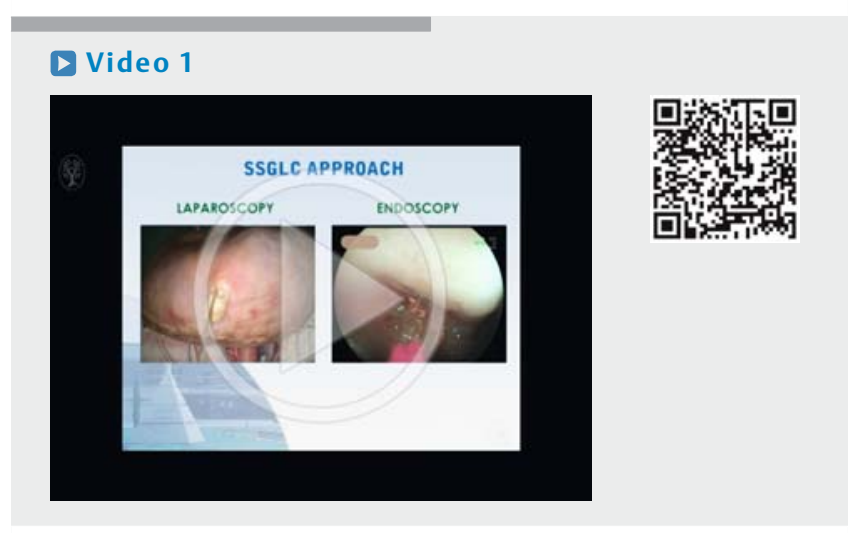

- Video 1: The safe scarless gastric laparoscopic closure (SSGLC) technique, combining the use of needlescopic instruments with a barbed suture during laparoscopically assisted transgastric hybrid natural orifice transluminal endoscopic surgery (NOTES). 

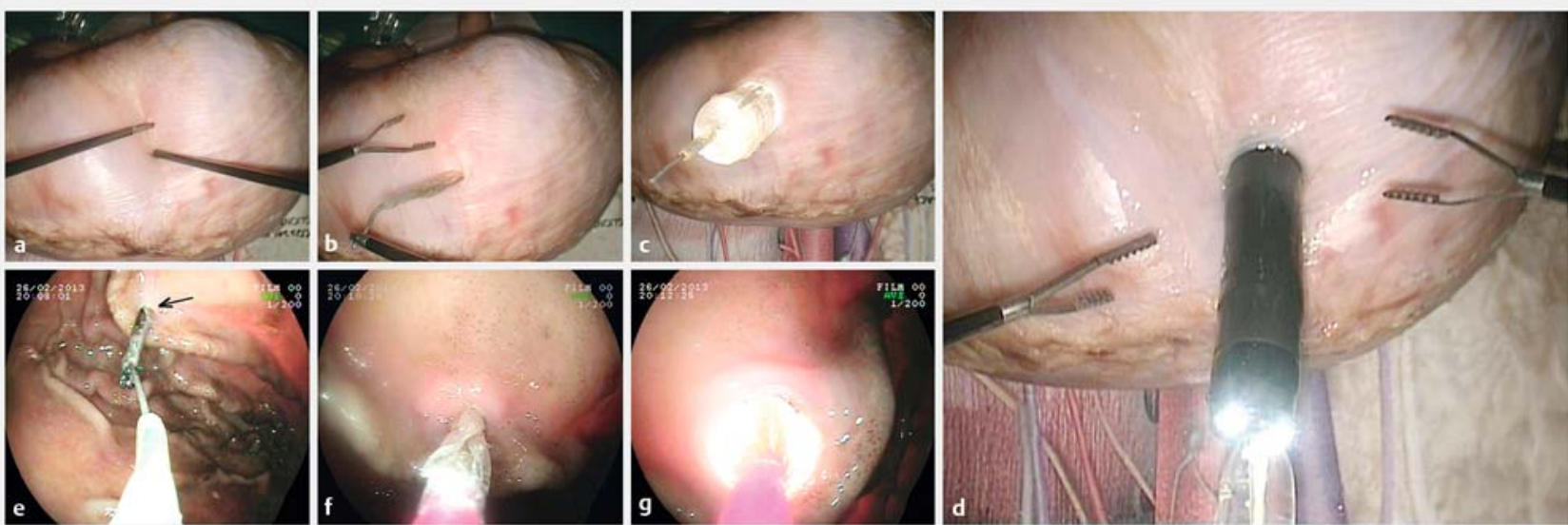

d

- Fig. 2 Endoscope entry maneuver, assisted by percutaneous needlescopic instruments, in a safe scarless gastric laparoscopic closure (SSGLC) procedure. Sequence of laparoscopic views (upper images and right-hand image) and endoscopic views (lower images). Note that no needleknife probe was needed. Bottom left image: the arrow shows the tip of the Minilap grasping the guidewire.
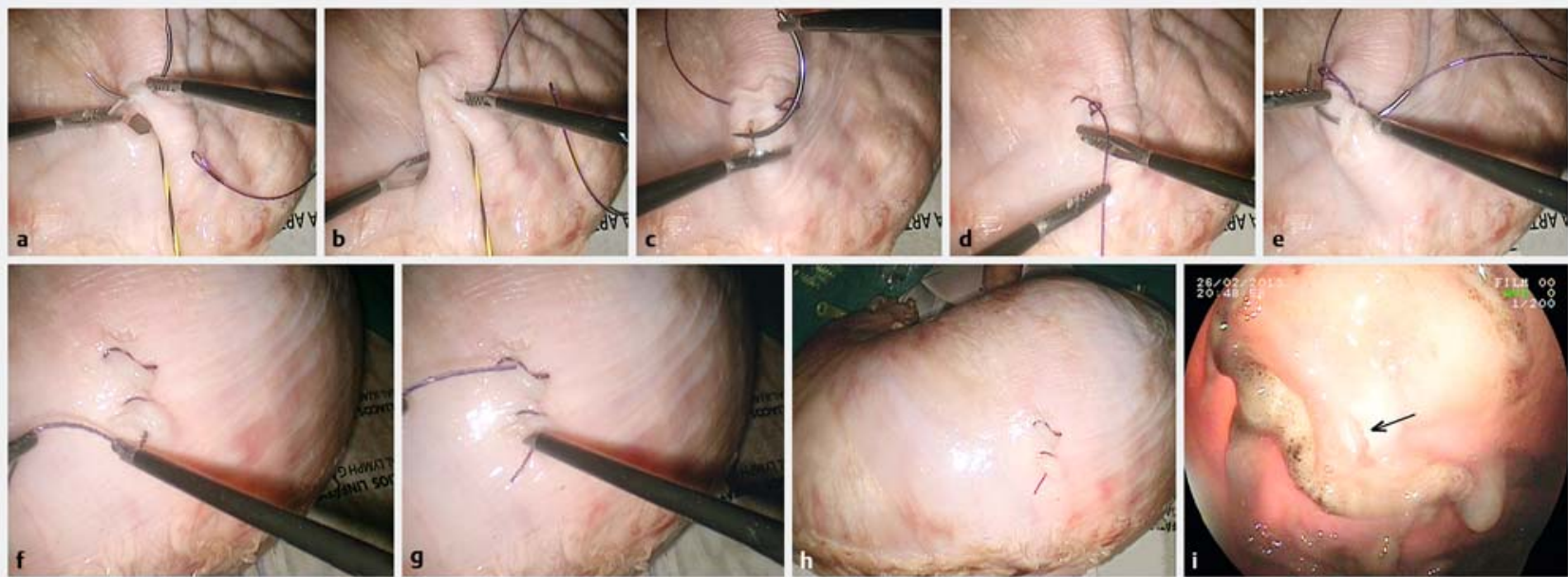

- Fig. 3 Sequence of safe scarless gastric laparoscopic closure (SSGLC), from the needlescopic plus V-Loc gastric closure to the "no-scissors" thread cut assisted by the MiniLap bevel tip. Combined final laparoscopic/endoscopic view, checking for the absence of leaks. The arrow shows invaginated gastric mucosa.

neously under laparoscopic surveillance ( $\vee$ Fig. 3). The total median (interquartile range $[\mathrm{IQR}])$ time for gastric closure was $16.7(10.05-23.3) \mathrm{min}$ ( $\mathbf{F i g . 4})$. Stomachs were inflated up to a pressure of $20 \mathrm{mmHg}$, using a carbon dioxide laparoscopic insufflator under electronic control; the maintenance of this pressure suggested the absence of gas leaks. Also, no air leaks were detected when the stomachs were submerged in water. A total of 6 experienced laparoscopic surgeons evaluated the procedure: 4/6 (67\%) considered SSGLC to be a safe and useful alternative to conventional laparo- scopic suture, and $6 / 6(100 \%)$ gave the maximum score for SSGLC feasibility and reproducibility. In addition, 2/6 (33\%) considered it to be as difficult to perform as conventional laparoscopic suturing, while 2/6 (33\%) found it easier (2/6 after adequate practice with a simulation trainer.

A gastric suture with serosal apposition [3], the incisionless and scarless features of needlescopic instruments [4], the safety of barbed sutures [5], and the ease of performance are the main advantages of this closure technique for hybrid NOTES.
Endoscopy_UCTN_Code_TTT_1AO_2AN

Competing interests

None 


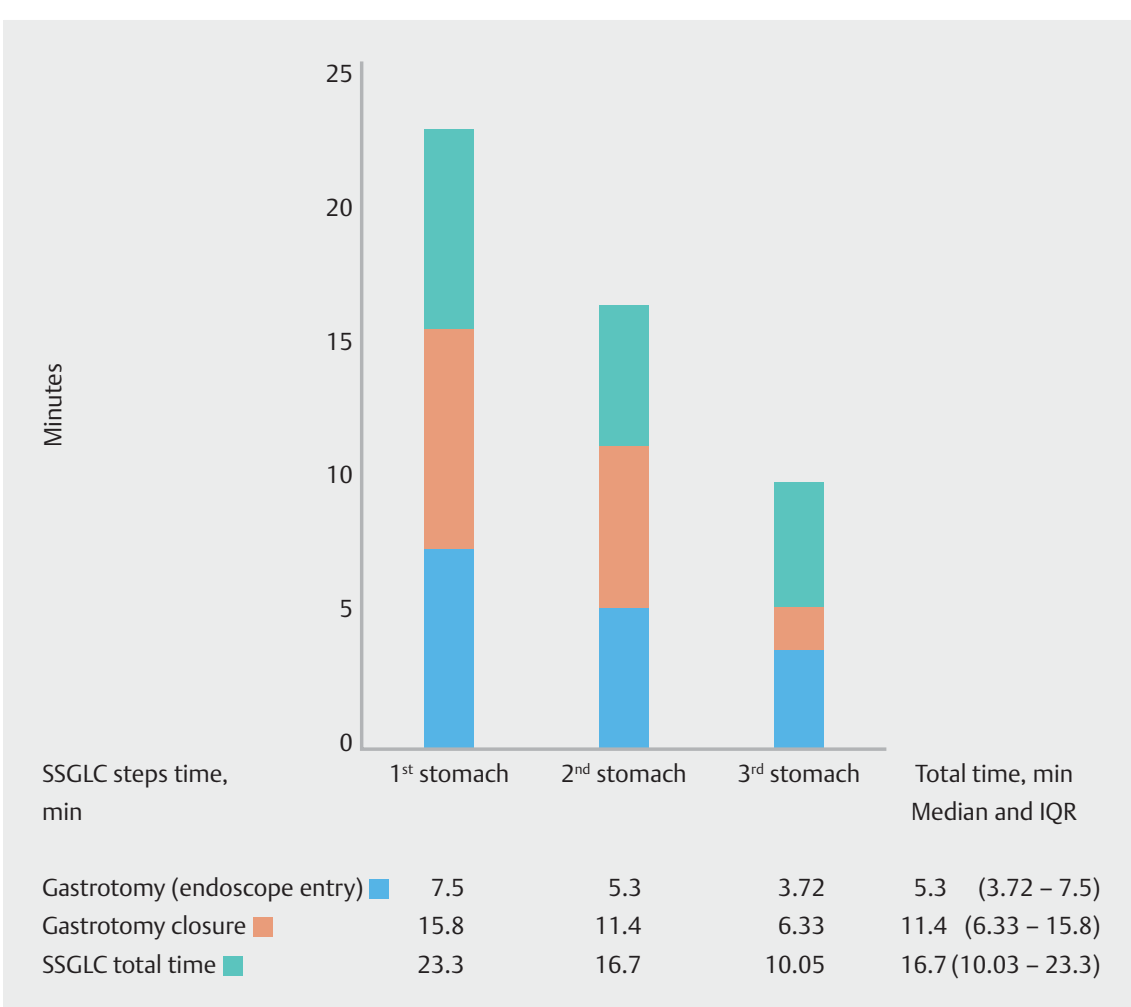

- Fig.4 Total and partial median times taken to perform safe scarless gastric laparoscopic closure (SSGLC) procedure.

\section{The Authors}

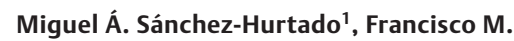
Sánchez-Margallo ${ }^{1}$, Jesús Usón-Casaús ${ }^{2}$, Alejandro Escribano-Crespo ${ }^{3}$, I. Díaz-Güemes Martín-Portugués ${ }^{1}$, Eva M. Pérez-Merino ${ }^{2}$

1 Laparoscopy Department, Jesús Usón

Minimally Invasive Surgery Centre, Cáceres, Extremadura, Spain
Corresponding author

Miguel Á. Sánchez-Hurtado, DVM, PhD Laparoscopy Department, Jesús Usón Minimally Invasive Surgery Centre, Ctra. N-521, km. 41,8 Cáceres, Extremadura, Spain

Fax: +34-927-181033

mhurtado@ccmijesususon.com

\section{References}

[1] Atallah S, Martin-Perez B, Keller D et al. Natural-orifice transluminal endoscopic surgery. Br J Surg 2015; 102: e73 - e92

[2] Tsin DA, Colombero LT, Lambeck J et al. Minilaparoscopy-assisted natural orifice surgery. JSLS 2007; 11: $24-29$

[3] Voermans RP, Worm AM, van Berge Henegouwen $\mathrm{Ml}$ et al. In vitro comparison and evaluation of seven gastric closure modalities for natural orifice transluminal endoscopic surgery (NOTES). Endoscopy 2008; 40: $595-601$

[4] Ross HM, Philp MM. Future directions in minimally invasive surgery. In: Ross HM, Lee S, Mutch MG, eds., et al. Minimally invasive approaches to colon and rectal disease. New York: Springer; 2015: 399-406

[5] Pacifico F. The barbed suture in bowel stapled anastomoses. Surgery Curr Res 2015; 5: 219

\section{Bibliography}

DOI http://dx.doi.org/10.1055/s-0043-103408

Endoscopy 2017; 49: E138-E140

(c) Georg Thieme Verlag KG

Stuttgart · New York

ISSN 0013-726X 